\title{
Mechanisms of aural encoding: I. Distinctive features for consonants'
}

\author{
R. A. COLE 2 , RALPH NORMAN HABER, AND B. D. SALES \\ UNIVERSITY OF ROCHESTER
}

To examine the importance of distinctive features that are used to encode consonants (following Wickelgren's analysis) in an immediate recall task, sequences of 5 consonants, all paired with the vowel /a/ were constructed and presented aurally for recall. The middle three items in each sequence all had either the same place of articulation (front, middle, or back of the vocal apparatus), or the same manner of articulation (voiced, unvoiced, or nasal), or were unrelated in either place or maner (control). It was shown that, in comparison with the control sequences, consonants imbedded among others articulated similarly were recalled less accurately, suggesting that these distinctive features are important in encoding and memory maintenance. A comparison of the 3 manner and 3 place features showed that the greatest difficulty in recall occurred for the similar manner sequences (especially voiced and unvoiced, implicating manner of articulation as the critical distinctive feature in aural encoding. Some discussion is also presented of a distinction between articulation and acoustic factors in encoding processes.

Studies measuring errors in short-term memory have found that stimuli which sound alike are more apt to be confused with each other in a serial recall task. While these experiments show the importance of acoustic factors in short-term memory, they cannot pinpoint the mechanism of encoding since the usual stimuli used have both acoustic and articulatory features in common. Thus, in a string such as B D C T P G, the letters all have the sound " $E$, " and the tongue always glides toward the same position. There can either be an acoustic representation of the stimulation in the nervous system, or an encoding from feedback of articulatory movements, or some combination of these.

Psychologists have tended to treat this distinction in an "either-or" manner, assuming either an acoustic or articulatory mechanism of aural encoding. It has long been known, however, that there is a close correspondence between the articulation of a sound and the acoustic makeup of that sound. The invention of the sound spectograph has enabled acoustic phoneticians to measure precisely the acoustic parameters of a sound. In Fig. 1, taken from Liberman (1957), stimuli in the same row have an identical manner of articulation, while stimuli in the same column have an identical place of articulation. The dark bars in each box are concentrations of energy at certain frequencies representing vowel resonance. The height of the "body" of each bar is identical because each consonant is paired with the same vowel, while the height and slope of the pointed end of the bar represent the transition cue by which consonants can be identified. This figure has been included here in order to show the very close correspondence between acoustic parameters and articulation. Going across any row, one can see that there is an identical acoustic parameter (the bottom formant) and an identical manner of articulation. Going down any column, there is an identical acoustic parameter (the top formant) and an identical place of articulation. Since there is such a close correspondence between the acoustic and articulatory parameters, it is impossible to devise stimuli which differ on only one of these dimensions, while the other is held constant.

There are several possible approaches by which to proceed in order to examine the encoding process. One converging operation has been to present white noise to the $S$ during rehearsal. White noise should act to prevent $S$ from "hearing" the sound rehearsed, but should not interfere with articulatory movements. When this condition has been employed (e.g., Hintzman, 1965; Murray, 1965), white noise does not interfere with performance. While this result supports a stress on an articulatory mechanism of encoding, Sperling (1963) reports that Ss hear the noise outside of their head, while rehearsal was going on inside of their head. Thus, white noise may be an ambiguous or ineffective converging operation, since $S$ may

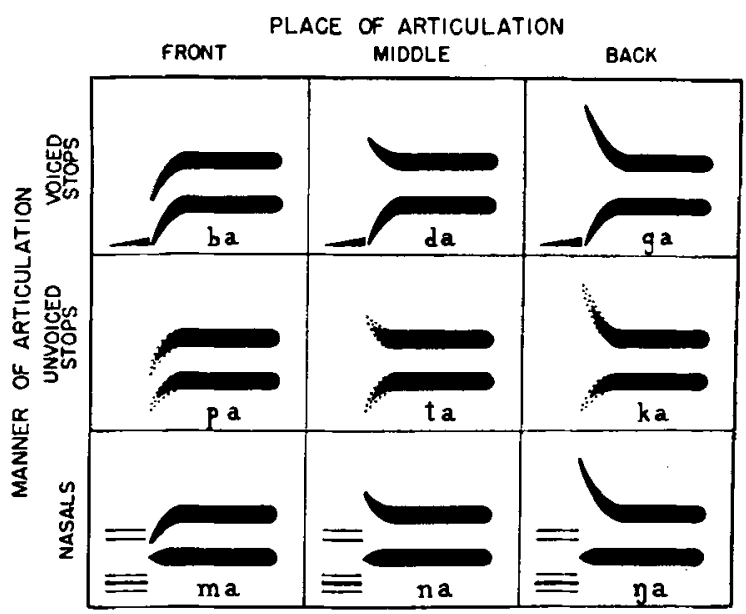

Fig. 1. Spectographic pattems that illustrate the transition cues for a stop and nasal consonants in initial position with the vowel $/ a /$. The dotted portions in the second row indicate the presence of noise (aspiration) in place of hamonics (taken from Liberman. 1957). 
handle it as an ignored channel (e.g., Broadbent, 1958). It should be possible to prevent $S$ from ignoring it, such as by requiring him to monitor the amplitude of the white noise by means of a pointer while he is encoding and rehearsing the material for recall. If recall was still unimpaired, the articulatory as compared to the acoustic factor would be further indicated as the rehearsal mechanism.

A reverse procedure, one to permit acoustic encoding but not the articulatory responses, might be to prevent $S$ from rehearsing, but during the encoding and rehearsal time, have $S$ hear a rehearsal in his earphones. He could be prevented from rehearsing by assigning him some other task during the period. This type of design is replete with problems, and to our knowledge has not been tried yet, but it does represent a possibility.

A third approach does not attempt to converge directly on a test between articulatory or acoustic mechanisms of encoding. As an example, Wickelgren (1966) studied intrusion errors for English consonants when all such stimuli were paired with the same vowel sound. He found that intrusion errors could be accurately predicted from a linguistic distinctive feature system in terms of voicing, nasality, place of articulation, and openess of the vocal tract. His results suggest that sounds are encoded into shortterm memory as a set of specific features, and a forgotten stimulus will be replaced by one with the most similar features.

The present experiment employs a related approach. If Wickelgren is correct, then it should be possible to construct sequences of consonants for recall in which specific features can be embedded in contexts of differing amounts of confusability. Thus, if voicing is an important feature in encoding and rehearsal, Ss should have great difficulty encoding a sequence in which all of the consonants are voiced. By comparing sequences which differ in the feature held in common, it should be possible to order the relative importance of these features as used in encoding and rehearsal.

The stimuli were chosen from Fig. 1. Since the columns differed only in the place of articulation, the rows only in the manner of articulation, sequences were constructed that placed consonants together from the same row, or the same column.

\section{METHOD}

\section{Subjects}

Fifty undergraduate University of Rochester students served as volunteer Ss. They were tested individually in a session lasting less than one hour.

\section{Stimuli}

A series of sequences of five consonants, each of which was paired with the vowel "a," were selected from among the nine consonants in Table 1. They were presented by a Wollensak tape recorder to each $\mathrm{S}$ seated in a small room with the $\mathrm{E}$. They were recorded by the first author who has had training in linguistics and in pronunciation of phones. They were recorded so that they were heard at a rate of one per second. Seven different types of sequences were used, differing according to the rules of selection of the items from Fig. 1. In the three different manner of articulation conditions, the second third, and fourth sound in each sequence was the same manner of articulation. Looking at Fig. 1 this means that the stimuli from the same row were in these positions. The sounds on either side of the sequence had neither place nor manner similar to the adjacent sound. Such a sequence for voiced consonants would be TA, BA, DA, GA, NA. In the three different place of articulation conditions the second, third, and fourth stimuli in each sequence had the same place of articulation. In reference to Fig. 1, this means that the stimuli were from the same column. The end positions were neither place nor manner of articulation similar to the adjacent sounds. Such a sequence would be NA, BA, PA, MA, GA. In the control condition, no sound in any of the positions had either place nor manner of articulation similar to adjacent sounds. Such a sequence would be $B A$, TA, GA, NA, PA. For each of the seven conditions six sequences were used, by constructing six sets of five sounds meeting these rules (except for the control condition, which had 18 sequences). Thus, each $\mathrm{S}$ heard 54 sequences. Within each condition of six sequences the sounds were balanced over positions. No sound was repeated within a sequence. Each $S$ received the 54 sequences in the same order, which was constructed from a random selection of the seven conditions.

\section{Procedure}

The $S$ was instructed that he would hear a list of five sounds. After the last one he should wait 3 sec and then report them in the order he heard them. During the report he was told that he would have as much time as he needed. He was told to rehearse the sound as he heard it and not just try to remember the consonant letter. The Ss' responses were tape recorded. For the basic analyses, the $\mathbf{S}$ was scored as correct for each sound in a sequence only if he named the proper sound in the proper position.

\section{RESULTS}

Figure 2 shows the accuracy of recall of the nine sounds for the control sequences, in which any interference due to similarity should be minimized. The differences observed are statistically significant ( $p$ $<.001$ ).

To determine the effects of similarity on recall, each sound in each of the three middle positions and in each condition was compared to the same sound in the respective position of the control se- 


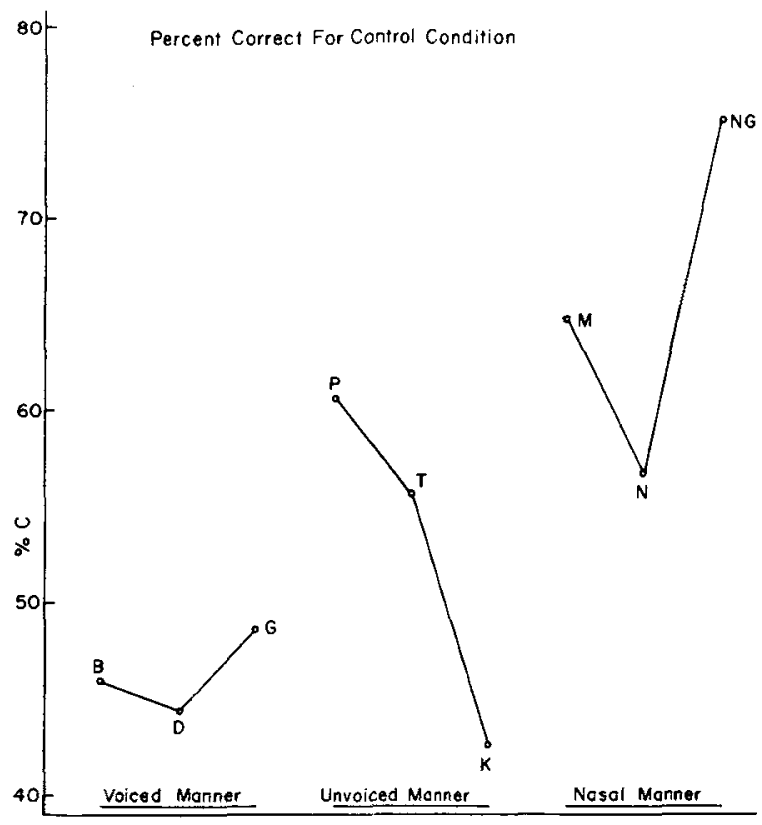

Fig. 2. Percentage of correct recall responses for each of the nine sounds used in the control sequences. Each point is summed over fifty $S s$, and for each $S$, is summed over all positions.

quences. The percentage correct for each sound was determined and then subtracted from the comparable score from the control sequences. Thus, for a particular sound in a particular condition, a positive number implies that the sound was easier to recall in the control condition than in a context of similar sounds. The scores for the three sounds defining each condition were combined, and these are shown in Fig. 3. An analysis of variance showed a main effect for position $(p<.001)$, indicating that position three produced the greatest differences between the control and the six experimental sequences. Individual $t$ tests indicated that only the nasal manner sequences dif-

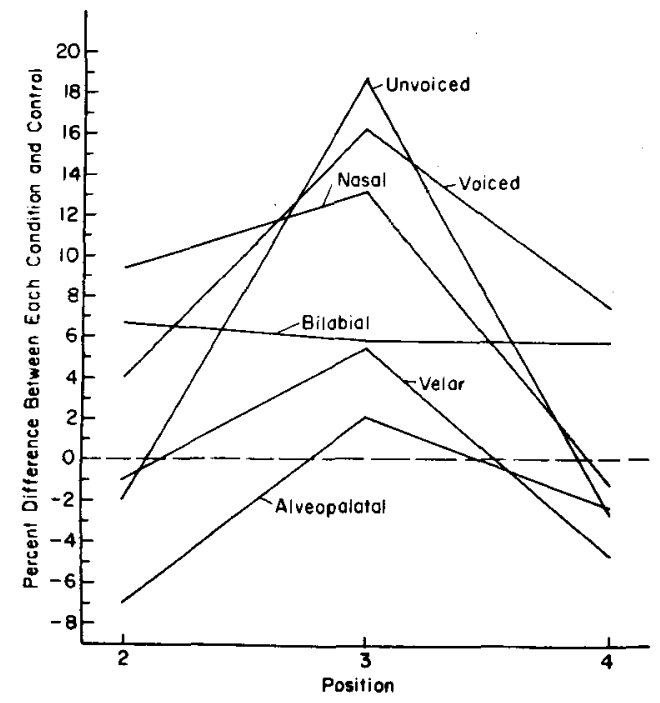

Fig. 3. Percentage differences between each condition and the comparable sounds appearing in the control sequences, as a function of position. Data are summed over $50 \mathrm{Ss}$. fered from the control in position two; none did in position four, while all three of the manner sequences did in position three (voiced, voiceless, and nasal). These latter comparisons suggested an interaction between position and condition which in fact was significant in the analysis of variance $(p<.01)$. A main effect of condition $(p<.05)$ indicated that the three manner conditions were generally more affected in all three positions by similarity of context than were the three place conditions. This was also supported by the pattern of $t$ test findings.

\section{DISCUSSION}

This experiment demonstrates: (1) it is possible to create interference in short-term memory by embedding sounds with similar distinctive features in the same context; (2) the context effect is greatest for the middle sounds, with little or no effect for the end sounds in the context; and (3) contexts that have similar manner of articulation create greater interference than contexts of similar place of articulation.

The interference effect shown in this study gives a powerful support to Wickelgren's notion that sounds are encoded in short-term memory as a set of features. It has now been shown that a distinctive feature hypothesis accurately predicts intrusion errors in short-term memory, and features show significant interference when sounds with similar features are embedded in the same context. The interference effect when similar features are used is a more powerful demonstration of encoding mechanisms than confusions in gross strings such as B C P T D in which one may phenomenally ldentify the " $E$ " sound in all stimuli. In sequences used in this experiment, one need be familiar with phonetics in order to point out the similar features.

The amount of interference seems unrelated to the overall ease of recalling the sound in a nonconfusable context. Thus in Fig. 1 (the control sequences) MA $\mathrm{NA}$ and NGA are easier to recall than BA DA and $\mathrm{GA}$, but there is more interference created when MA NA and NGA are placed together in the same sequence.

It appears as if distinctive features involving manner of articulation (voicing, lack of voicing, nasality) are more critical in the encoding and rehearsal process than are ones of place of articulation. Since this study has no means of separating out the distinctive features due to articulation from the distinctive features due to acoustic characteristics, it is still not known whether it is the distinctiveness involved in speaking the sounds, or in hearing them that is critical in the encoding process. A different design is needed to answer this critical question.

\section{References}

Broadbent, D. E. Perception and communication. New York: Pergamon Press, 1958. 
Hintzman, D. L. Classification and aural coding in short-term memory. Psychon. Sci., 1965, 3, 161-162.

Liberman, A. M. Some results of research on speech perception. J. Acoust. Soc. Amer., 1957, 29, 117-123.

Murray, D.J. The effect of white noise upon the recall of vocalized lists. Canad, J. Psychoi., 1965, 19, 333-345.

Sperling, G. A model for visual memory tasks. Hum. Factors, $1963,5,17-31$.

Wickelgren, W. A. Distinctive features and errors in short-term memory for English consonants. J. Acoust. Soc. Amer., 1966, 39, 388-398.

\section{Notes}

1. This research was supported in part by a grant from the United
States Public Health Service MH 10753, and by a grant from the National Science Foundation GB 2909 , to the second author, and by the Undergraduate Research Participation Program grant to the Psychology Department of the University of Rochester from the National Science Foundation GY 2619 in an award to the first author. We would like to thank Barbara Herr for her assistance in data collection and analysis, Audrey Konow for computer programming, and Robert Kulikowski for his participation in running the experiment.

2. Now at the University of California at Riverside.

(Accepted for publication October 9, 1967.)

Abstract: Structure in perceptual classification by S. Imai, Hokkaido University, and W. R. Garner, Yale University

An article was recently published as a Psychonomic Monograph Supplement that should be of interest to readers of this Journal. It was published as a Monograph because it was a long article and because space in this Journal is under heavy pressure, but an abstract of it is given below so that readers will know of its existence and may either purchase it (price $\$ 1.00$ ) or refer to it in their libraries. Imai, S. and Garner, W. R. Structure in perceptual classification. Pyschon. Monogr. Suppl., 1968, 2, No. 9 (Whole No. 25):

Four experiments were carried out on the role of structure of stimulus sets in perceptual classification. All experiments used a total set of nine visual stimuli derived from three forms times three colors. The first experiment showed that the judged similarity of any pair of stimuli depended only on whether the pair differed by one or by two attributes; thus there was in effect no metric structure of the attributes. The second experiment required $S$ to select a single subset from the total set so as to include different stimuli specified by E. This experiment showed that the structure of the subset itself and of the total set both influenced how subsets were selected. The third experiment required selections of two subsets, again so as to include different stimuli specified by $E$. This experiment showed that the structure of the two subsets combined is similar to the structure of selected single subsets. The fourth experiment required complete classification (partitioning) of subsets of 4, 5, or 6 different stimuli selected by $\mathrm{E}$. Regardless of the size or structure of a subset, classification tended to maintain the attribute structure of the total set. Taken together, these experiments demonstrate that attribute structure of the total set and subsets is more important in determining perceptual classification than simple similarity and difference relations between stimuli. Furthermore, there is the strong suggestion that subsets or classes are perceived more in relation to larger classes and the total set than in contrast to other classes of the same size. 